\title{
Toward a Synthetic Acoustic Ecology: Sonically Situated, Evolutionary Agent Based Models of the Acoustic Niche Hypothesis
}

\author{
Alice Eldridge $^{1,2}$ and Chris Kiefer ${ }^{1}$ \\ ${ }^{1}$ Emute Lab, Department of Music, University of Sussex, UK \\ ${ }^{2}$ Peck Labs, Evolution, Behaviour \& Environment, University of Sussex, UK \\ alicee@sussex.ac.uk
}

\begin{abstract}
We introduce the idea of Synthetic Acoustic Ecology (SAC) as a vehicle for transdisciplinary investigation to develop methods and address open theoretical, applied and aesthetic questions in scientific and artistic disciplines of acoustic ecology. Ecoacoustics is an emerging science that investigates and interprets the ecological role of sound. It draws conceptually from, and is reinvigorating the related arts-humanities disciplines historically associated with acoustic ecology, which are concerned with sonically-mediated relationships between human beings and their environments. Both study the acoustic environment, or soundscape, as the literal and conceptual site of interaction of human and non-human organisms. However, no coherent theories exist to frame the ecological role of the soundscape, or to elucidate the evolutionary processes through which it is structured. Similarly there is a lack of appropriate computational methods to analyse the macro soundscape which hampers application in conservation. We propose that a sonically situated flavour of Alife evolutionary agent-based model could build a productive bridge between the art, science and technologies of acoustic ecological investigations to the benefit of all. As a first step, two simple models of the acoustic niche hypothesis are presented which are shown to exhibit emergence of complex spectro-temporal soundscape structures and adaptation to and recovery from noise pollution events. We discuss the potential of SAC as a lingua franca between empirical and theoretical ecoacoustics, and wider transdisciplinary research in ecoacoustic ecology.
\end{abstract}

\section{Introduction}

There is increasing interest across ecological science (Pijanowski et al. 2011, Sueur and Farina, 2015), arts (Barclay and Gifford 2018, Monacchi and Krause, 2017; McCormack et al., 2009) and humanities (Turner et al., 2003) in listening to, recording, investigating and interpreting the acoustic environment - or soundscape - as the interface of human and natural systems. If bioacoustics can be characterised as the study of the isolated duets of vocalising critters, then the nascent field science of ecoacoustics is concerned with investigating and interpreting the ecological relevance of the strains of full orchestra. A similar shift is evident in the arts as representation in field recordings and music has broadened from individual voices to collective creature choruses as more powerful, informative narratives in soundscape composition (Monacchi, 2013; Barclay and Gifford, 2018). However, there is a paucity of coherent theory addressing the ecological significance of global soundscapes, lack of effective computational tools for ecological monitoring (Sueur et al. 2008) and many latent creative applications, for example in musical composition or game world design. Just as Alife modelling has potential to mediate theoretical and empirical biology (Wheeler et al. 2002), we propose that a sonically situated flavour of Alife, which we call Synthetic Acoustic Ecology (SAC), may be a productive vehicle for investigation and a nexus of exchange between science, art and technological facets of acoustic ecology in advancing our appreciation of soundscape as an interface of human and natural systems which both reflects and affects our coupled environments.

\section{Soundscape, Ecoacoustics and Acoustic Ecology}

The term 'soundscape' has been used by a variety of disciplines to describe the relationship between a landscape and the composition of its sound in both real and virtual worlds (Grimshaw and Schott, 2007). Originally coined in the context of urban design (Southworth, 1967) Soundscape was later used by a group of environmentally-aware radio artists and sonic sociologists to describe 'the acoustical characteristics of an area that reflect natural processes' (Schafer. 1977). The scientific discipline of soundscape ecology recently proposed a framework to investigate soundscape in terms of the causes and consequences of biological (biophony), geophysical (geophony), and human-produced (anthrophony) sounds that emanate from a landscape (Pijanowski et al. 2011). The emerging interdisciplinary science of Ecoacoustics subsumes both soundscape ecology and bioacoustics (Sueur and Farina, 2015) to study the ecological role of sound. There is a growing impetus to develop acoustic ecology as a truly interdisciplinary endeavour (Barclay and Gifford, 2018), bridging traditional disciplinary divides. This is where we position Synthetic Acoustic Ecology.

Whereas bioacoustics infers behavioural information from intra- and interspecific signals, ecoacoustics inves- 
tigates the ecological role of sound at higher ecological and evolutionary organisational units - from population and community up to landscape scales. Sound is understood as a core ecological component (resource) and ipso facto, due to structuring by competition, an indicator of ecological status (source of information). The field has been substantially bolstered by the increasing availability and decreasing costs of automated recording devices (Acevedo and VillanuevaRivera, 2006, Farina et al. 2018), cheap storage and developments in acoustic data processing (Truskinger et al. 2014). However, whilst it has drawn from theories of related ecological disciplines including bioacoustics, and landscape ecology (Turner et al. 2001), there is an absence of coherent theory regarding the ecological significance of the macro soundscape. This not only constrains theoretical advances, but hampers potential applications such as environmental monitoring and prediction. Ecoacoustics is born of Bioacoustic Big Data, but lacks coherent theories and computational tools for effective development and application.

\section{Acoustic Niche Hypothesis}

Three hypotheses underpin ecoacoustics. The morphological adaptation hypothesis (MAH) and the acoustic adaptation hypothesis (AAH) are borrowed by bioacoustics and describe how signals evolve through ecological feedback; the acoustic niche hypothesis is core to ecoacoustics (and is also the hardest to evaluate) and describes the evolution of soundscape complexity. The MAH focuses on the 'sender' and hypothesises that the embodied form (body size, trachea length, beak shape etc.) will shape potential range of signals (Bennet-Clark 1998). The AAH (Morton, 1975) predicts that acoustic properties of an environment can influence the evolution of vocalizations in certain species.

In his formulation of the ANH, musician-turned bioacoustician Bernie Krause pointed out that both morphological and behavioural adaptations can also be triggered by interspecific interference when organisms' calls contain similar frequency and timing features (Krause, 1993). The ANH was proposed after observation of complex arrangements of non-overlapping signals in recordings of soundscapes across multiple habitats. Krause postulated that this could be explained by evolutionary pressure to minimize spectral or temporal overlaps in interspecific vocalizations. The ANH expands Hutchinson's ecological niche concept (Hutchinson, 1957) by adding a sonic dimension to evolutionary ecospace. That vocalising species partition acoustic space to minimise interference from sympatric species has long been recognised (Duellman and Pyles, 1983) in bioacoustics. As illustrated in Figure 1 frequency partitioning across major taxa is common in tropical biomes, including frogs (Amézquita et al., 2011), although the theory has also been challenged (Chek et al. 2003).

The more significant and controversial prediction follows that soundscape structure is a proxy for ecological integrity:

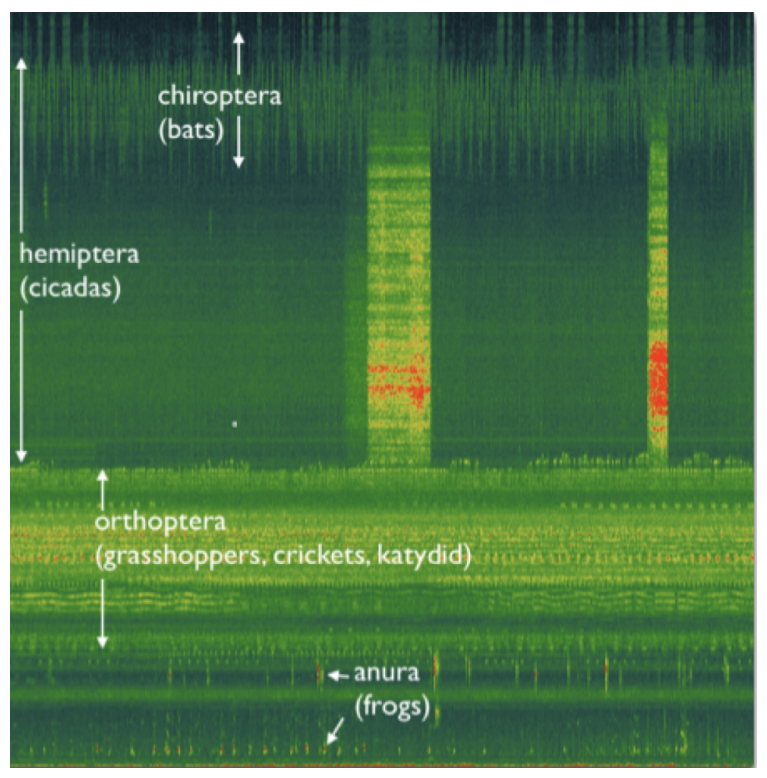

Figure 1: Spectrogram $(0-22.5 \mathrm{kHz})$ of a field recording made in the Ecuadorian amazon showing inter-taxon frequency partitioning of the acoustic environment. The vocalisations of each taxa are bandlimited, minimising frequency overlap between species; quasi temporal partitioning can also be observed in anuran species, although it is not clear here if this is conspecific or heterospecific.

less-disturbed habitats with unaltered species assemblages will exhibit higher levels of coordination between interspecific vocalizations than more heavily disturbed habitats, where species assemblages are in rapid flux. Likewise, invasive species could create biophonic disturbances, thereby altering natural acoustic partitioning (Pijanowski et al., 2011). This implies that if we listen in the right way we can hear the health of an ecosystem.

ANH is foundational to ecoacoustic theory and has major implications for ecological monitoring and prediction, however empirical validation and development of application is hard: firstly because it is not clear exactly what it means for a soundscape to exhibit 'higher levels of coordination'; secondly because measurement of ecological integrity and even biodiversity remains contentious (Hillebrand et al. 2018); thirdly we lack appropriate computational methods for community-level machine listening (Eldridge et al. 2016). Whilst research into bioacoustically motivated machine listening algorithms for automated species detection is well developed (e.g. Stowell and Plumbley (2014)), community level indices are less well developed. Recent research evaluating community acoustic indices against classical biodiversity measures in marine (Harris et al. 2016) and terrestrial (Sueur et al., 2014) habitats are promising, but new research directions are needed. Thus empirical theory testing is hampered by lack of requisite computational technology, 
but computational development requires clearer conceptual models and empirical methods. Modelling provides a complementary mode of investigation to potentially break this impasse by stimulating new, empirically testable, questions and exploring computational acoustic metrics.

\section{Ecoacoustic Perspectives in Performance}

Technical and conceptual inspiration also comes from related creative practices. Research and practice in ecosystem based sound art and computer music has shared theoretical foundations with Alife and a history of dialog across these disciplines. For example, Waters (2007) conceptualises music as complex dynamical interaction within an ecosystem of performer, instrument and environment, emphasising the aesthetic value of emergence. Earlier work in Alife and sonic ecosystems (McCormack, 2003) demonstrated the artistic potential of agent based evolutionary systems with implicit fitness through competition for resources, eliminating the fitness bottleneck of more conventional evolutionary models. The value of emergent complexity in ecosystem based music is emphasised further by Bown (2009), Eigenfeldt and Pasquier (2011) and in work such as Di Scipio's Audible Eco-Systemic Interface (Di Scipio, 2003). Principles and methods developed through these works are carried through in the models presented in this paper.

\section{Towards a Synthetic Acoustic Ecology}

The potential for agent-based evolutionary modelling to investigate the $\mathrm{ANH}$, and advance understanding of the interactions between soundscape, agents and environment is patent, but as yet unexplored. Rich cross-disciplinary interactions are afforded as software models can productively draw upon insights from generative experimental music and machine listening research to both develop ecological applications and in turn to feed back into creative practice.

Whilst standard symbolic models may provide a platform to investigate ecological and musical implications of the acoustic niche hypothesis in the abstract, we propose that much more can be gained through a commitment to emergent, embodied and situated models á la Alife. A commitment to Emergence (rather that explicitly simulating higher levels of soundscape structure) is critical to ecoacoustic investigation because we are interested in understanding how these macro behaviours arise from the interactions of vocalising organisms with each other via their environment (both physical and the acoustic environment to which they contribute and adapt). It is exactly this scaling up from bioacoustic investigation of reciprocal individual communication (signal-receiver) model to understanding the complex, messy dynamics of acoustic communities which ecoacoustics seeks to expound. And the reason for paucity of theoretical frameworks methods. Sonic Situatedness means direct implementation in digital audio rather via symbolic representations and a commitment to implement listening and vocalising via digital audio analysis and synthesis methods. These could be run offline, but also allows for realtime audio processing through which the model interacts with real-world acoustic environment on biological-critterlike time scales. Sonic embodiment therefore requires a microphone and speaker (and attendant digital-analogue converters). As well as embodying key properties of the phenomena of study, this sonically situated approach develops in silico - in vivo models, which interface with complex realities they seek to understand; we believe this may be of value as a vehicle for cross-disciplinary exchange and application at the intersects of technological and biological scientific and artistic enquiry

\section{Models and methods}

We implement a sonically situated agent-based evolutionary model of the ANH. The ANH implies a direct form of ecological inheritance, as in classical niche construction (Day et al. 2003). However, whereas the activity of one species may create an ecological opportunity for another species in other dimensions of ecospace (for example, fish thriving upstream of beaver dam) the acoustic environment is a shared, finite resource: acoustics activity of one species is at best irrelevant (if outside perceptual range - think bats and elephants) and often in competition between species.

Two models were built. The aim of model 1 was to establish the minimal conditions for the emergence of spectrotemporal partitioning through low level agent-environment competitive interactions. Model 2 investigated the impact of adding greater variation in agent temporal and frequency parameters on complexity of agent calling behaviours and soundscape structure; the response of the population to externally induced environmental sound, simulating noise pollution, was also investigated. Source code and example outputs from these models are available online (Eldridge and Kiefer, 2018).

\section{Model 1: Simple Acoustic Niche Partitioning}

The basic premise of the acoustic niche hypothesis was tested: that interference from heterospecific signals will result in spectro-temporal partitioning of the shared acoustic environment. A synchronous evolutionary model was implemented using asexual reproduction, where each agent can be seen as a proto-species. Agents exist in a non-spatiallyexplicit, acoustic world; they all hear each other equally. Model audio is calculated at a sample rate of $44.1 \mathrm{kHz}$ with floating point accuracy. A timestep $t$ represents a period of 512 audio samples.

Genome and state Each agent is genetically specified by a frequency $(f)$ and phase $(\theta)$ which determine both vocalising and listening behaviours; agent state is represented by an energy level $\epsilon$. 
Agent vocalisation and hearing Agents vocalise at time step $t$, if $t \bmod \eta=\theta$, where $\eta$ is a global constant. When vocalising, they emit a sine tone for 512 samples at frequency $f$ (at one of 96 possible pitches, linearly spaced between $200 \mathrm{~Hz}$ and $9800 \mathrm{~Hz}$ ) and universally defined amplitude. As observed in biological species (e.g. (Amézquita et al. 2011) ) frequency range of hearing $f \pm \beta$ is wider than vocalising frequency, where $\beta$ is a fixed, universal amount. Because we are interested in population-level effects, bioacoustic notions of 'sender' and 'receiver' are consolidated into one asexual critter: Vocalising agents also 'listen' in the same time step by calculating the value

$$
\mu_{i}=\frac{\sum_{f-\beta}^{f+\beta}\left(g-a_{i}\right)}{2 \beta}
$$

where $g$ is a vector of spectral magnitudes of the global soundscape, and $a_{i}$ is a vector containing the spectrum of the vocalisation of an agent $i . \mu_{i}$ is used to calculated an energy change $\epsilon_{i}+\delta_{i}$ as follows:

$$
\delta_{i}= \begin{cases}C_{\text {comms }} & \text { if } \mu_{i}<T, \\ -X_{\text {over }} & \text { if } \mu_{i}>T_{\text {over }} \\ -X_{\text {masked }} & \text { otherwise }\end{cases}
$$

If $\mu_{i}$ is above fixed threshold $T$, communication is considered to be masked and the agent loses energy (tax); if it is below $T$ vocalising is potentially successful and agent accrues energy. If $\mu_{i}$ is above a higher threshold $T_{\text {over }}$ then the area of the spectrum is considered to be overcrowded and the agent is taxed more heavily. Thresholds, energy credits and taxes are fixed and constant across the population.

Agent behaviour and fitness assignment Just as in wild ecosystems (rather than cattle or pigeon or optimisation tasks), fitness is defined implicitly (McCormack, 2003), relative to the current state of the environment. All agents vocalising at timestep $t$ are taxed by amount $X_{v o x}$, representing the energy that is needed to create sound. These vocalisations are mixed and written to a global soundscape buffer which is readable by all agents and stored as a cumulative record of population evolution. At each timestep, a Fast Fourier Transform (FFT) of the global soundscape is calculated (FFT size: 512, hop size: 512), consisting of the sum of all agent vocalisations, and any additional environmental sound (see below).

Asexual reproduction and death When any agent energy $\epsilon_{i}$ reaches a defined threshold $T_{\text {repro }}$, asexual reproduction may occur with probability $P_{\text {spawn }}$. A fixed reproductive tax $X_{\text {repro }}$ is imposed and a single offspring is introduced into the population. $\theta$ are creep mutated with wrap-around with a fixed probability in a uniform distribution $P_{m u t}$. Mutation changes are drawn from a normal distribution, and scaled by
$S_{m u t} . f$ is mutated with a lower probability $P_{m u t_{f}}$ reflecting more considerable physiological changes necessary for changes in vocalisation pitch (Bennet-Clark, 1998). Every timestep, agents with $\epsilon_{i} \leq 0$ are removed from the population.

Initialisation and parameters The population was initialised with $N_{p o p}$ individuals; gene values are drawn randomly from uniform distributions and initial energy values $\epsilon_{i}$ are drawn from a normal distribution $(\mu: 220, \sigma: 50)$. Figure 2 shows a typical run of the model over 200,000 timesteps, parameterised as follows: $\beta: 20, \eta: 100$, $X_{\text {vox }}: 5, X_{\text {masked }}: 5, X_{\text {repro }}: 50, X_{\text {over }}: 250$, $C_{\text {comms }}: 17, T_{\text {repro }}: 300, T:-6.1, T_{\text {over }}:-5.13$, $P_{\text {mut }}: 0.1, P_{\text {muf }}: 0.05, S_{\text {mut }}: 0.15, N_{\text {pop }}: 500$. Parameter values were experimentally determined to afford longterm stability of population size.

\section{Model 2: Introducing variation in vocal complexity and perceptual acuity}

The second model investigates the impact of greater variation in agent vocalisation and listening behaviours on the complexity of the resultant soundscape.

Genome and state In addition to frequency $(f)$ and phase $(\theta)$, agents in this model have an expanded genome to represent hierarchical temporal structures with shifting periodicity, as observed in the syllables and phrases of birds, frogs and bat species (Bohn et al. 2008). Vocalisation is dependent on the following conditions at timestep $t$ :

$$
t \bmod \eta_{i}=\theta \wedge \operatorname{seq}(t)
$$

where $\eta_{i}$ is individual periodicity rather than a global constant as used in model 1 , within the range $\left[0, \eta_{\max }\right]$, and $\operatorname{seq}(t)$ is a function that divides time into sections (equivalent to musical bars) or length $\eta_{i}$, and chooses whether a vocalisation occurs during that section, according to a variable length pattern bit pattern. It is defined as follows:

$$
\operatorname{seq}(t)=\left(2^{\left\lfloor\frac{t}{\eta_{i}}\right\rfloor \bmod \Theta_{\text {len }}} \wedge \Theta_{\text {pattern }}\right)>0
$$

$\Theta_{l e n}$ defines the length of the sequence (measured in sections or bars), in the range $\left[1, \Theta_{\max }\right]$. $\Theta_{\text {pattern }}$ defines the pattern of the sequence. For example, if $\Theta_{\text {pattern }}$ is equal to $100_{2}$ then an agent will vocalise every three bars.

Vocalisations still occur at a single genetically encoded frequency $(f)$, but recognition bandwidth $\beta$ is also evolvable between global limits $\beta_{\min }$ and $\beta_{\max }$. To accompany this change, threshold $T$ is expanded to a threshold range between $T_{l o}$ and $T_{h i}$. For each agent, $T$ is calculated between these global limits to conserve overall 'area' of spectral energy-magnitude within which vocalisations are judged to be successful or masked. This results in agents with 
high $\beta$ having a better opportunity of reproducing, balancing against the implicit advantage for agents with low $\beta$. Agent behaviour, energy assignment, and reproduction are identical to model 1. With these new additions, the genome in model 2 contains $\left\{f, \theta, \eta, \beta, \Theta_{\text {len }}, \Theta_{\text {pattern }}\right\}$.

Initialisation and parameters The model is initialised as model 1, with the following additions: $T_{l o}:-5.87, T_{h i}$ : $-5.36, \beta_{\min }: 20, \beta_{\max }: 50, \eta_{\max }: 100, \Theta_{\max }: 8$.

Analyses Model performance was evaluated by examining i) the evolution of population-level soundscape structures and ii) population-level adaptation to externally induced noise pollution. Both full models were further compared to null modes in which agent phenotypes (time-frequency parameters of their vocalisations) were randomised at each time step, rather than genetically determined.

Quantitative evaluation of niche separation is an open problem. On the assumption that niche formation leads to structuring of soundscape, established complexity metrics were adopted as a proxy for evaluation: spectral entropy (SE) (Kapucu et al. 2016) and Lempel-Ziv Complexity (LZC) (Lempel and Ziv, 1976). SE is a measure of the complexity or regularity of the frequency dynamics of a signal; LZC measures the randomness and harmonic variability of signals. LZC works in the binary domain; following Aboy et al. (2006), each spectral frame was thresholded at the median to create binary input sequences. Both metrics were calculated from sequential 100-frame averages of the spectrogram soundscape, reflecting the maximum small-scale vocalisation periodicity that was permitted in both models.

Population adaptivity was evaluated by simulating interference from noise pollution. After allowing the population to stabilise (after 40,000 iterations, or approximately $8 \mathrm{~min}$ utes), a short burst of maximum amplitude, white noise, low pass filtered at $2 \mathrm{kHz}$ was added to the global soundscape, and the ability of the population to recover observed.

\section{Results}

Spectrograms for both models (figures 2, 37 show soundscapes that evolve distinct frequency bands with simple temporal patterns emerging. Both also show population recovery following simulated noise pollution: agents in mid to lower frequency bands die out due to masking from white noise; the population gradually recovers to create a soundscape exhibiting full-range spatio-temporal structures. Population recovery is evident from plots of population size (Fig. 4): both models 1 and 2 are robust to noise pollution, the null model populations become extinct.

Figure 5 shows spectrograms of the first and last minutes of each model in more detail. In the first minutes, model 1 makes a smooth transition from randomness to visible structure, while the agents in model 2 rapidly die off, presumably due to stronger competition of resources reflected in the wider listening bandwidth $\beta$, also observable in changes in population size (figure 4). In the final minute, strong frequency partitioning with regular cyclic patterns are evident in model 1, due to the fixed global $\beta$ and $\eta$. Model 2 evolves similar frequency partitioning, but a sparser and more varied structure due to the more flexible methods of agent vocalisation.

As can be seen in figure 6. SE values vary dramatically between null models and those in which vocalisations are evolved. For both full models, SE quickly drops from an initial high value, reflecting the formation of structure from the initial random population. The value then rises again when filtered white noise hits. When noise ceases, and only high frequency vocalising agents are left in the world, SE drops then slowly rises and stabilises as new spectrotemporal structures form. In null model $1 \mathrm{SE}$ drops as the population dies out, creating a simplification of structure; in null model 2 SE remains high, reflecting the lack of structure in the small, random population.

Figure 7 shows the results of LZC analysis for both models and their nulls. For both full models, LZC drops from an initial high value as structure forms from the initial random population, and roughly stabilises in the longer term at a much lower value. In the null models we see a similar pattern to SE: null 1 drops due to population extinction; null 2 maintains a constant value, reflecting a lack of structure.

\section{Discussion}

Our toy models demonstrate that spectro-temporal partitioning, creating structured global soundscapes, emerge readily from sonically situated agent-environment interactions. This partitioning emerges both from random populations, and from a small population recovering from masking by noise pollution. That small increases in vocalising and listening capacities lead to quite significant increases in soundscape variation lends credence to this line of enquiry. The spectrogram for model 2 (figure 3 ) shows variation in vocalising strategies reminiscent of different taxa, and the audio files reveal phasing reminiscent of the shifting densities of tropical anuran choruses.

We also acknowledge that these models are a proof of concept and need to be more rigorous and grounded in order to be of scientific value. For example, the agents only emit single frequencies and communicate synchronously. A more realistic model would support vocalisations of arbitrary complexity, and agent memory to allow asynchronous call and response behaviours. Given the number of free parameters, we are mindful of WYWIWYG (what you want is what you get) (Wheeler et al. 2002), although we try to mitigate by beginning with a minimal model. The models do however capture the core characteristics of spectro-temporal soundscape partitioning, and SE and LZC metrics show a formation of structure, laying the ground for more rigorous work in SAC in the future. 


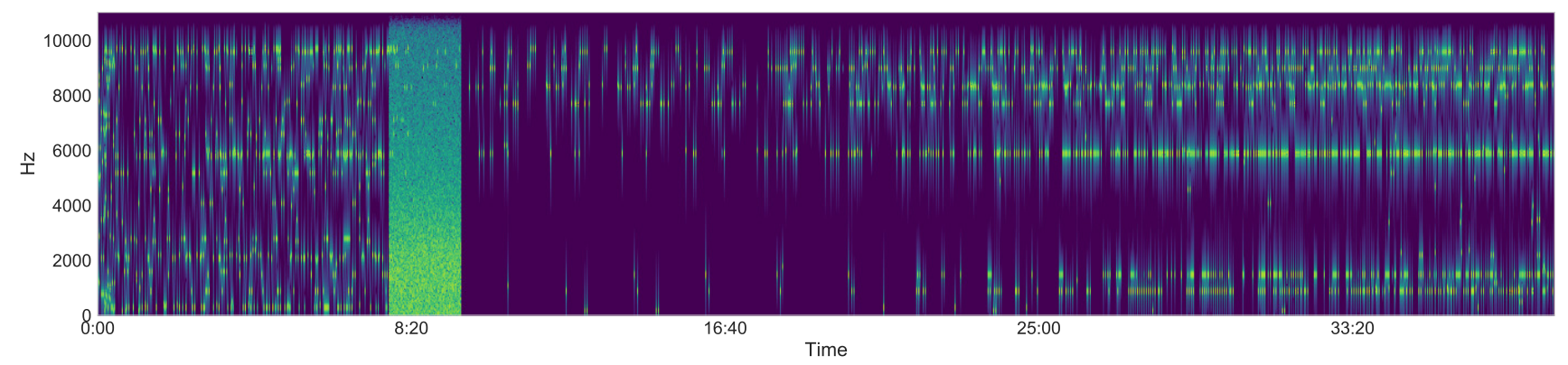

Figure 2: Spectrogram of the world soundscape over a typical run of model 1 seeded with a population of agents with randomly initialised gene and energy values.

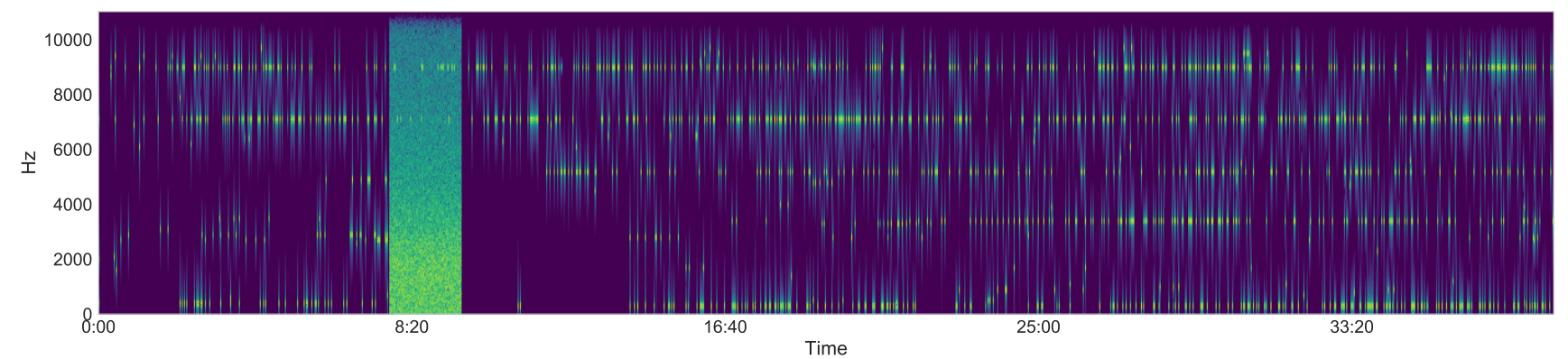

Figure 3: Spectrogram of the world soundscape over a typical run of model 2 seeded with a population of agents with randomly initialised gene and energy values.

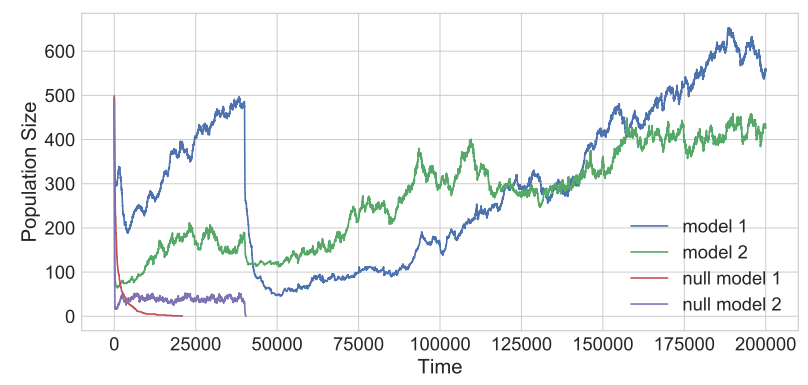

Figure 4: Population size for all models. Filtered white noise was introduced at iteration 40,000 .

\section{Synthetic Acoustic Ecology as a Lingua Franca for a Transdisciplinary Acoustic Ecology}

These models illustrate the sonically situated principle of the proposed SAC, where interaction between agents and the world take place through digital audio, drawing upon methods and principles of computer music and machine listening. Just as perceptually situated Alife models Alife afford exploration of emergent phenomena without regressing to high level explanatory theories, sonically situated models offer a means to explore soundscape as an emergent phenomena.

Back in 2001, Seth Bullock suggested that Alife models might serve as a lingua franca between empirical and theoretical biology (Wheeler et al. 2002). Bullock suggests Alife simulations can open up dialogue between those fol- lowing formal modelling approaches and the wider biology community, under the conditions that these models are both scientifically rigorous and communicable with pedagogical transparency and clarity - 'completely rigourous and maximally luminous'. We should also be careful to avoid projecting added reality onto simulated models. Our sonically situated models output digital audio which is isomorphic in format with empirical and creative soundscape data; they can can be auditioned and viewed as spectrograms and analysed using same machine listening techniques as artistic and scientific acoustic ecology research. Although our models are simple, the framework arguably represents a literal lingua franca, not only between empirical and theoretical ecoacoustics, but across artistic and scientific, cultural and biological enquiries concerned with listening to, recording, analysing and interpreting the soundscape. SAC supports theoretical investigation to understand how soundscape are shaped by and shape agent behaviours, and also conceivable may foster insights into novel machine listening methods for population-level soundscape analyses which could bolster application in ecological monitoring and prediction.

\section{Future developments}

The models presented here provide a proof of concept, and an initial support for the basic premises of the ANH. Obvious immediate developments include integration of biologically-plausible models of vocalisations of organisms from different taxa, development of agent morphologies and 

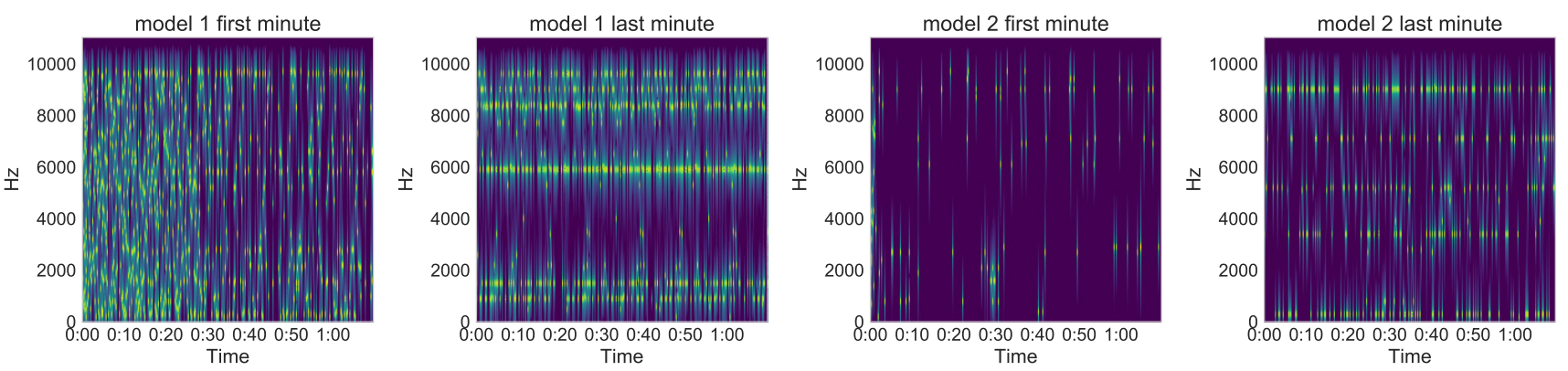

Figure 5: Zooming in: first and final minutes for each model

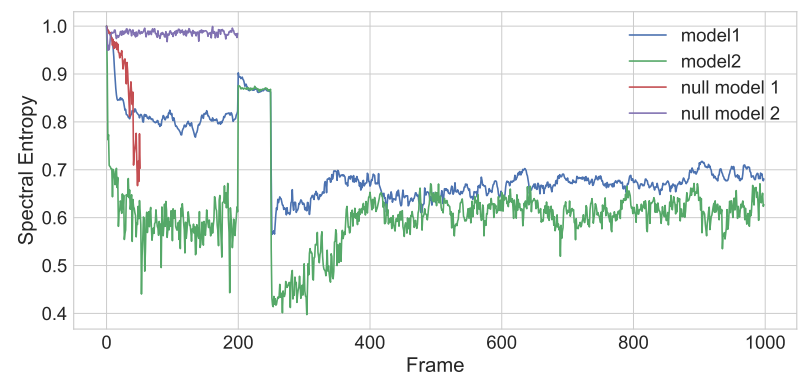

Figure 6: SE, measured over averages of 100 FFT frames

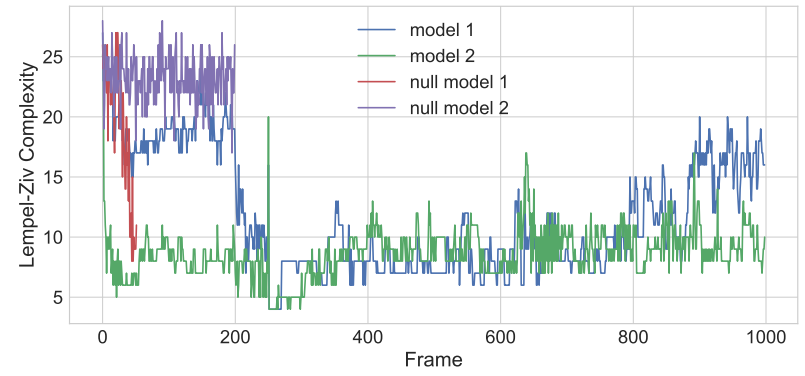

Figure 7: LZC, measured over averages of 100 FFT frames

spatial dimensionality. Beside theoretical explication, we see potential applications in both planning and creative contexts: agent-based modelling could contribute to growing research which aims to mitigate the impact of noise pollution in planned marine development (Codarin et al. 2009) and land-use changes (e.g. airport expansion, shipping channels etc.); intriguing compositional prospects also exist for application in procedurally generated soundscape synthesis in VR and game worlds, a similarly vibrant area of research and development (Yannakakis and Togelius, 2017).

\section{Conclusion}

We introduce Synthetic Acoustic Ecology as a fecund vehicle of enquiry for, and exchange between, scientific and artistic investigation within the wider field of acoustic ecology. Two proof-of-concept models of the Acoustic Niches Hypothe- ses were presented which demonstrated emergent spectrotemporal soundscape partitioning due competition between sonically situated agents. We highlight the potential of SAC as a prospective lingua franca for empirical and theoretical ecoacoustics, and wider artistic, technical and scientific exchange, which could lead to genuinely transdisciplinary frameworks for the investigation of the acoustic environment as the interface of human and natural systems.

\section{References}

Aboy, M., Hornero, R., Abásolo, D., and Álvarez, D. (2006). Interpretation of the lempel-ziv complexity measure in the context of biomedical signal analysis. IEEE Transactions on Biomedical Engineering, 53(11):2282-2288.

Acevedo, M. A. and Villanueva-Rivera, L. J. (2006). Using automated digital recording systems as effective tools for the monitoring of birds and amphibians. Wildlife Society Bulletin, 34:211-214.

Amézquita, A., Flechas, S. V., Lima, A. P., Gasser, H., and Hödl, W. (2011). Acoustic interference and recognition space within a complex assemblage of dendrobatid frogs. Proceedings of the National Academy of Sciences, 108(41):1705817063.

Barclay, L. and Gifford, T. (2018). The art and science of recording the environment. Leonardo, 51:184-184.

Bennet-Clark, H. (1998). Size and scale effects as constraints in insect sound communication. Philosophical Transactions of the Royal Society of London B: Biological Sciences, 353(1367):407-419.

Bohn, K. M., Schmidt-French, B., Ma, S. T., and Pollak, G. D. (2008). Syllable acoustics, temporal patterns, and call composition vary with behavioral context in mexican free-tailed bats. The Journal of the Acoustical Society of America, 124(3):1838-1848.

Bown, O. (2009). Ecosystem models for real-time generative music: A methodology and framework. In in Proceedings of the 2009 International Computer Music Conference (ICMC 2009. Citeseer.

Chek, A. A., Bogart, J. P., and Lougheed, S. C. (2003). Mating signal partitioning in multi-species assemblages: a null model test using frogs. Ecology Letters, 6(3):235-247. 
Codarin, A., Wysocki, L. E., Ladich, F., and Picciulin, M. (2009). Effects of ambient and boat noise on hearing and communication in three fish species living in a marine protected area (miramare, italy). Marine pollution bulletin, 58(12):18801887.

Day, R., Laland, K., and Odling-Smee, J. (2003). Rethinking adaptation the niche-construction perspective. Perspectives in Biology and Medicine, 46(1):80 - 95.

Di Scipio, A. (2003). 'sound is the interface': from interactive to ecosystemic signal processing. Organised Sound, 8(3):269277.

Duellman, W. E. and Pyles, R. A. (1983). Acoustic resource partitioning in anuran communities. Copeia, pages 639-649.

Eigenfeldt, A. and Pasquier, P. (2011). A sonic eco-system of selforganising musical agents. In European Conference on the Applications of Evolutionary Computation, pages 283-292. Springer.

Eldridge, A., Casey, M., Moscoso, P., and Peck, M. (2016). A new method for ecoacoustics? toward the extraction and evaluation of ecologically-meaningful soundscape components using sparse coding methods. PeerJ, 4:e2108.

Eldridge, A. and Kiefer, C. (2018). Supplementary materials for 'Toward a Synthetic Acoustic Ecology: Sonically Situated Evolutionary Agent-Based Models of the Acoustic Niche Hypothesis'. https://doi.org/10.5281/zenodo. 1252326

Farina, A., Gage, S. H., and Salutari, P. (2018). Testing the ecoacoustics event detection and identification (eedi) approach on mediterranean soundscapes. Ecological Indicators, 85:698715 .

Grimshaw, M. and Schott, G. (2007). Situating gaming as a sonic experience: the acoustic ecology of first person shooters. In Situated Play: Proceedings of DIGRA, Digital Games Research Association, pages 91-98. DIGRA.

Harris, S. A., Shears, N. T., and Radford, C. A. (2016). Ecoacoustic indices as proxies for biodiversity on temperate reefs. Methods in Ecology and Evolution, 7(6):713-724.

Hillebrand, H., Blasius, B., Borer, E. T., Chase, J. M., Downing, J. A., Eriksson, B. K., Filstrup, C. T., Harpole, W. S., Hodapp, D., Larsen, S., et al. (2018). Biodiversity change is uncoupled from species richness trends: consequences for conservation and monitoring. Journal of Applied Ecology, 55(1):169-184.

Hutchinson, G. (1957). Concluding remarks.: Cold spring harbor symposia on quantitative biology.

Kapucu, F. E., VÃlkki, I., Mikkonen, J. E., Leone, C., Lenk, K., Tanskanen, J. M. A., and Hyttinen, J. A. K. (2016). Spectral entropy based neuronal network synchronization analysis based on microelectrode array measurements. Frontiers in Computational Neuroscience, 10:112.

Krause, B. (1993). The niche hypothesis, a hidden symphony of animal sounds, the origins of musical expression and the health of habitats. The Explorers journal, Winter:156-160.

Lempel, A. and Ziv, J. (1976). On the complexity of finite sequences. IEEE Transactions on information theory, 22(1):75-81.
McCormack, J. (2003). Evolving sonic ecosystems. Kybernetes.

McCormack, J., Eldridge, A., Dorin, A., and McIlwain, P. (2009). Generative algorithms for making music: emergence, evolution, and ecosystems. In Dean, R., editor, The Oxford Handbook of Computer Music. Oxford University Press.

Monacchi, D. (2013). Fragments of extinction: acoustic biodiversity of primary rainforest ecosystems. Leonardo Music Journal, pages 23-25.

Monacchi, D. and Krause, B. (2017). Ecoacoustics and its expression through the voice of the arts: An essay. Ecoacoustics: The Ecological Role of Sounds, pages 297-312.

Morton, E. S. (1975). Ecological sources of selection on avian sounds. The American Naturalist, 109(965):17-34.

Pijanowski, B. C., Villanueva-Rivera, L. J., Dumyahn, S. S., Farina, A., Krause, B., Napoletano, B. M., Gage, S. H., and Pieretti, N. (2011). Soundscape ecology: the science of sound in the landscape. BioScience, 61(3):203-216.

Schafer, R. (1977). The Soundscape: Our Sonic Environment and the Tuning of the World. Destiny Books, Rochester, Vermont.

Southworth, M. F. (1967). The sonic environment of cities. $\mathrm{PhD}$ thesis, Massachusetts Institute of Technology.

Stowell, D. and Plumbley, M. D. (2014). Automatic large-scale classification of bird sounds is strongly improved by unsupervised feature learning. PeerJ, 2:e488.

Sueur, J. and Farina, A. (2015). Ecoacoustics: the ecological investigation and interpretation of environmental sound. Biosemiotics, 8(3):493-502.

Sueur, J., Farina, A., Gasc, A., Pieretti, N., and Pavoine, S (2014). Acoustic indices for biodiversity assessment and landscape investigation. Acta Acustica united with Acustica, 100(4):772-781.

Sueur, J., Pavoine, S., Hamerlynck, O., and Duvail, S. (2008). Rapid acoustic survey for biodiversity appraisal. PloS one, 3(12):e4065.

Truskinger, A., Cottman-Fields, M., Eichinski, P., Towsey, M., and Roe, P. (2014). Practical analysis of big acoustic sensor data for environmental monitoring. In Big Data and Cloud Computing (BdCloud), 2014 IEEE Fourth International Conference on, pages 91-98. IEEE.

Turner, M. G., Gardner, R. H., O'neill, R. V., et al. (2001). Landscape ecology in theory and practice, volume 401. Springer.

Turner, P., McGregor, I., Turner, S., and Carroll, F. (2003). Evaluating soundscapes as a means of creating a sense of place. Georgia Institute of Technology.

Waters, S. (2007). Performance ecosystems: Ecological approaches to musical interaction. EMS: Electroacoustic Music Studies Network, pages 1-20.

Wheeler, M., Bullock, S., Paolo, E. D., Noble, J., Bedau, M., Husbands, P., Kirby, S., and Seth, A. (2002). The view from elsewhere: Perspectives on alife modeling. Artificial life, 8(1):87-100.

Yannakakis, G. N. and Togelius, J. (2017). Artificial Intelligence and Games. Springer. 